\title{
DNA topoisomerase II $\alpha$ (TOP2A) inhibitors up-regulate fatty acid synthase gene expression in SK-Br3 breast cancer cells: In vitro evidence for a 'functional amplicon' involving FAS, Her-2/neu and TOP2A genes
}

\author{
JAVIER A. MENENDEZ ${ }^{1,2}$, LUCIANO VELLON ${ }^{1,2}$ and RUTH LUPU ${ }^{1,2}$ \\ ${ }^{1}$ Department of Medicine, Evanston Northwestern Healthcare Research Institute, Evanston, IL; \\ ${ }^{2}$ Department of Medicine, Northwestern University Feinberg School of Medicine, Chicago, IL, USA
}

Received August 17, 2006; Accepted September 18, 2006

\begin{abstract}
Fatty acid synthase (FAS), the key metabolic multi-enzyme that is responsible for the terminal catalytic step in the de novo fatty acid biosynthesis, plays an active role in the development, maintenance, and enhancement of the malignant phenotype in a subset of breast carcinomas. We recently described that a molecular bi-directional cross-talk between FAS and the Her-2/neu (erbB-2) oncogene is taking place at the level of transcription, translation, and activity in breast cancer cells. Because Her-2/neu has been linked with altered sensitivity to cytotoxic drugs, we envisioned that FAS gene expression may represent a novel predictive molecular factor for breast cancer response to chemotherapy in a Her-2/neurelated manner. We herein evaluated whether chemotherapyinduced cell damage acts in an epigenetic fashion by inducing changes in the transcriptional activation of FAS gene in breast cancer cells. To evaluate this option, FAS- and Her-2/neuoverexpressing SK-Br3 breast cancer cells were transiently transfected with a FAS promoter-reporter construct (FASLuciferase) harboring all the elements necessary for high level expression in cancer cells. SK-Br3 cells cultured in the presence of topoisomerase II $\alpha$ (TOP2A) inhibitors doxorubicin and etopoxide (VP-16) demonstrated a 2- to 3-fold increase in FAS promoter activity when compared with control cells growing in drug-free culture conditions. We failed to observe any significant activation of FAS promoter following exposure to the anti-metabolite 5-fluorouracil, the alkylating drug cisplatin, or the microtubule interfering-agents paclitaxel
\end{abstract}

Correspondence to: Professor Ruth Lupu or Dr Javier A. Menendez, Evanston Northwestern Healthcare Research Institute, 1001 University Place, Evanston, IL 60201, USA

E-mail: r-lupu@northwestern.edu

E-mail: jmenendez@enh.org

Key words: fatty acid synthase, Her-2/neu, erbB-2, chemotherapy, topoisomerase II $\alpha$, DNA replication, breast cancer and vincristine. Moreover, the up-regulatory effects of TOP2A inhibitors on the transcriptional activation of FAS gene expression were not significantly decreased when the FAS promoter was damaged at the sterol regulatory element binding protein (SREBP)-binding site. Considering that FAS inhibition produces profound inhibition of DNA replication and S-phase progression in cancer cells, we finally asked whether a crosstalk between TOP2A and FAS could exhibit a Her-2/neurelated bi-directional nature. TOP2A protein levels were decreased during treatment with the anti-Her-2/neu antibody trastuzumab while, concomitantly, FAS promoter activity and FAS protein expression were significantly reduced. Of note, when the expression levels of TOP2A protein were analyzed following exposure of SK-Br3 cells to increasing concentrations of the novel slow-binding FAS inhibitor C75, a dose-dependent reduction in TOP2A expression was observed. Although FAS gene is not physically located in the Her-2/neuTOP2A amplicon, our present findings strongly suggest that a tight functional association between FAS, Her-2/neu and TOP2A genes is taking place in a subset of breast carcinoma cells.

\section{Introduction}

After numerous basic research and clinical studies, it now appears that fatty acid synthase (FAS), the key metabolic multienzyme that is responsible for the terminal catalytic step in the de novo fatty acid biosynthesis (1), may play a previously unrecognized role in the development, maintenance, and enhancement of the malignant phenotype of several malignancies including breast cancer (2). In fact, infiltrating carcinomas of the breast constitutively express high levels of FAS compared to non-transformed human epithelial tissues, while overexpression and hyperactivity of FAS is associated with more aggressive breast carcinomas and poor clinical outcomes (3-6). Furthermore, increased levels of FAS accompany the development of in situ carcinoma of the breast, suggesting a potential link between FAS up-regulation and increased risk of breast cancer development (7). Accordingly, FAS-dependent endogenous fatty acid biogenesis is a molecular feature that accompanies the malignant transformation of 
NIH-3T3 fibroblasts induced by Her-2/neu (erbB-2) oncogene, which is overexpressed not only in invasive breast cancer but also in premalignant atypical duct proliferations and in ductal carcinoma in situ of the breast (8). Moreover, FAS activity plays a necessary role in the oncogenic ability of Her-2/neu, including Her-2/neu-induced anchorage-independent cell growth and increased cell survival (8). It is reasonable to suggest that a molecular bi-directional cross-talk between FAS and Her-2/neu is taking place at the level of transcription, translation, and activity in breast cancer cells. In one hand, high levels of both FAS expression and activity positively correlate with Her-2/neu oncogene amplification and/or overexpression in a wide panel of human breast cancer cell lines (9), thus suggesting that FAS may be a novel down-stream effector of Her-2/neu-promoted tumorigenicity and breast cancer progression. Accordingly, a transcriptome analysis of Her-2/neu revealed that FAS is one of the genes differentially regulated by Her-2/nеu in human breast epithelial cells (10). On the other hand, pharmacological FAS inhibitors cerulenin and $\mathrm{C} 75$ have been found to suppress Her-2/neu oncoprotein expression in breast Her-2/neu overexpressors (11). Similarly, Her-2/neu expression is dramatically down-regulated when FAS gene expression is silenced by using the highly sequencespecific mechanism of RNA interference (RNAi). Pharmacological and RNAi-mediated inhibition of FAS represses Her2/neu expression at the transcriptional level, concomitantly up-regulating the expression of PEA3, an Ets factor that specifically reverses the in vitro transformed phenotype of Her2/neu-overexpressing cancer cells through the attenuation of the Her-2/neu oncogene promoter activity (11). These results, altogether, demonstrate that the response of breast cancer cells to metabolic stress after perturbation of FAS activity is accompanied by the specific suppression of Her-2/neu oncogene, which is amplified in 20-35\% of invasive breast cancers and is known to be associated with shortened diseasefree and overall survival (12). Therefore, Her-2/neu oncogene could act as a molecular sensor of energy imbalance that participates actively in the maintenance of an abnormally elevated endogenous fatty acid metabolism in breast cancer cells.

Pharmacological and/or small interference RNA-induced inhibition of FAS signaling synergistically sensitizes cultured breast cancer cells to anti-mitotic drugs such as docetaxel (Taxotere $^{\mathrm{TM}}$ ), vinorelbine (Navelbine ${ }^{\mathrm{TM}}$ ) and paclitaxel $\left(\right.$ Taxol $\left.^{\mathrm{TM}}\right)$ (13-15). Moreover, this FAS-related chemosensitization is also synergistic with the anti-Her-2/neu humanized monoclonal antibody trastuzumab (Herceptin ${ }^{\mathrm{TM}}$ ) (11). Because Her-2/neu has been linked with altered sensitivity to cytotoxic drugs according to in vitro studies and clinical trials (16), we recently envisioned that FAS gene expression may represent a novel predictive molecular factor for breast cancer response to chemotherapy in a Her-2/neu-related manner. Here, we evaluated whether chemotherapy-induced cell damage may act in an epigenetic fashion by inducing changes in the transcriptional activation of breast cancerassociated FAS gene. To evaluate this option, Her-2/neuoverexpressing SK-Br3 breast cancer cells were transiently transfected with a FAS promoter-reporter construct (FASLuciferase) that harbors all the elements necessary for high level expression in cancer cells. The SK-Br3 cells contain levels of FAS constituting up to $28 \%$, by weight, of the cytosolic protein (17). Remarkably, an increase in the rate of transcription of the FAS gene, and consequently a higher abundance of FAS mRNA, was found to be primarily responsible for FAS overexpression in this breast cancer cell line, whereas increases in FAS message stability and longer half-life of the FAS protein were not detected (18). Taken together, the experimental evidence makes SK-Br3 cells an ideal in vitro model system for studying the regulation of FAS gene. We reveal for the first time that topoisomerase II $\alpha$ (TOP2A) inhibitors doxorubicin and etopoxide (VP-16), but not other chemotherapeutic agents, work in an epigenetic fashion by up-regulating the activity of FAS gene promoter in SK-Br3 breast cancer cells. When the expression levels of TOP2A protein were analyzed following pharmacological blockade of FAS activity, a dose-dependent reduction in TOP2A protein expression was observed. These results not only suggest that a previously unrecognized bi-directional cross-talk between TOP2A and FAS seems to occur in Her2/neu-overexpressors but further reveal TOP2A as a good molecular candidate that actively participates in the linkage between FAS-catalyzed endogenous fatty acid metabolism and DNA replication in breast cancer cells.

Although the clinical and therapeutic importance of FAS expression status to breast cancer management should be resolved in clinical studies, the characterization of a putative 'functional amplicon' involving FAS, Her-2/neu and TOP2A genes should enhance our understanding of how FASdependent endogenous fatty acid metabolism, once considered a minor anabolic-energy-storage-pathway in normal cells, may be consider a 'metabolic oncogene' that actively contributes to the cancer phenotype through previously unexpected bi-directional cross-talks with well-characterized cancerregulating genes such as Her-2/neu and TOP2A.

\section{Materials and methods}

Materials. C75 was purchased from Alexis Biochemicals (San Diego, CA, USA). C75 was dissolved in dimethyl sulfoxide (DMSO) and stored in the dark as stock solution $(50 \mathrm{mg} / \mathrm{ml})$ at $-20^{\circ} \mathrm{C}$. For experimental use, stock solution was diluted with growth medium. In all cases, final concentrations of DMSO were $<0.1 \%$ and did not modify the proliferation of control cells.

The primary antibody for FAS immunoblotting was a mouse $\mathrm{IgG}_{1}$ FAS monoclonal antibody (clone 23) from BD Biosciences Pharmingen (San Diego, CA, USA). Anti-cerbB-2/HER-2/neu (phosphor-specific) Ab-18 (clone PN2A) mouse monoclonal antibody was from NeoMarkers (Lab Vision Corporation, Fremont, CA, USA). Anti-Human Topo II $\mathrm{AB}$ rabbit polyclonal antibody was from TopoGEN, Inc. (Columbus, OH, USA). Anti-ß-actin goat polyclonal antibody was from Santa Cruz Biotechnology, Inc. (Santa Cruz, CA, USA).

Cell lines and culture conditions. SK-Br3 breast cancer cells were obtained from the American Type Culture Collection (ATCC) and they were routinely grown in phenol redcontaining improved MEM (IMEM, Biosource International, Camarillo, CA, USA) supplemented with $5 \%(\mathrm{v} / \mathrm{v})$ fetal bovine 
serum (FBS) and $2 \mathrm{mM}$ L-glutamine at $37^{\circ} \mathrm{C}$ in a humidified atmosphere of $95 \%$ air and $5 \% \mathrm{CO}_{2}$, unless otherwise specificed.

FAS promoter activity. Using FuGENE 6 transfection reagent (Roche Biochemicals, Indianapolis, IN, USA) as directed by the manufacturer, overnight serum-starved cells seeded into 24-well plates $\left(\sim 5 \times 10^{4}\right.$ cells/well $)$ were transfected in lowserum $(0.1 \%$ FBS $)$ media with $300 \mathrm{ng} /$ well of the pGL3Luciferase (Promega, Madison, WI, USA) construct containing a luciferase reporter gene driven by either an intact (FAS wtSREBP-BS-Luc) or damaged (FAS $\triangle$ SREBP-BS-Luc) 178-bp FAS promoter fragment along with $30 \mathrm{ng} /$ well of the internal control plasmid pRL-CMV, which was used to correct for transfection efficiency. After $18 \mathrm{~h}$, the transfected cells were washed and then incubated in the absence or presence of chemotherapeutic agents as specified. Approximately $24 \mathrm{~h}$ after treatments, luciferase activity from cell extracts was detected using a Luciferase assay system (Promega) according to the protocol specified by the manufacturer in a VICTOR2 ${ }^{\mathrm{TM}} 1420$ multilabel counter (Perkin Elmer). The magnitude of activation in FAS promoter-Luciferase-transfected cells was determined after normalization to the luciferase activity in cells cotransfected with equivalent amounts of the empty pGL3luciferase vector lacking the FAS promoter ( $\varnothing$-Luciferase) and the internal control plasmid pRL-CMV, which was taken as 1.0-fold. This control value was used to calculate the relative (fold) change in transcriptional activities of FAS promoterLuciferase-transfected cells in response to treatments after normalization to pRL-CMV activity, and the data are shown as the means \pm SD from three separate experiments (performed in triplicate).

Immunoblotting. Cells were harvested by treatment with trypsin-EDTA solution, washed twice with PBS (-) and stored at $-80^{\circ} \mathrm{C}$. The cells were lysed in lysis buffer $[20 \mathrm{mM}$ Tris (pH 7.5), $150 \mathrm{mM} \mathrm{NaCl}, 1 \mathrm{mM}$ EDTA, $1 \mathrm{mM}$ EGTA, $1 \%$ Triton X-100, $2.5 \mathrm{mM}$ sodium pyrophosphate, $1 \mathrm{mM}$ ßglycerolphosphate, $1 \mathrm{mM} \mathrm{Na} \mathrm{VO}_{4}, 1 \mu \mathrm{g} / \mathrm{ml}$ leupeptin, $1 \mathrm{mM}$ phenylmethylsulfonylfluoride] for $30 \mathrm{~min}$ on ice, and then a particle-free supernatant solution was obtained by centrifugation at $14.000 \mathrm{x} \mathrm{g}$ for $15 \mathrm{~min}$. All operations were at $0-4^{\circ} \mathrm{C}$. A sample was taken for measurement of protein content by a BioRad assay (Bio-Rad Laboratories). Equal amounts of protein were heated in SDS sample buffer (Laemmli) for $10 \mathrm{~min}$ at $70^{\circ} \mathrm{C}$ and subjected to electrophoresis on $3-8 \%$ NuPAGE Tris-Acetate gels $\left(\right.$ Novex $\left.^{\mathrm{TM}}\right)$ and transferred to nitrocellulose membranes. Non-specific binding on the nitrocellulose filter paper was minimized by blocking for $1 \mathrm{~h}$ at room temperature (RT) with TBS-T [ $25 \mathrm{mM}$ Tris- $\mathrm{HCl}, 150 \mathrm{mM} \mathrm{NaCl}(\mathrm{pH} \mathrm{7.5)}$, and $0.05 \%$ Tween-20] containing $5 \%(\mathrm{w} / \mathrm{v})$ nonfat dry milk. The treated filters were washed in TBS-T and then incubated with primary antibodies for $2 \mathrm{~h}$ at RT in TBS-T containing 1\% (w/v) nonfat dry milk. The membranes were washed in TBS-T, horseradish peroxidase-conjugated secondary antibodies (Jackson Immuno Research, West Grove, PA) in TBS-T were added for $1 \mathrm{~h}$, and immunoreactive bands were visualized with ECL detection reagent (Pierce, Rockford, IL). Blots were reprobed with an antibody for B-actin to control for protein loading and transfer. Densitometric values of protein bands were quantified using Scion imaging software (Scion Corp., Frederick, MD, USA).

Statistical analyses. All observations were confirmed by at least three independent experiments. The data are presented as means \pm SD. The Student's t test (paired and unpaired) was used to evaluate the statistical significance of mean values. Statistical significance levels were $\mathrm{P}<0.05$ and $\mathrm{P}<0.005$. All Ps are two-tailed.

\section{Results}

Topoisomerase IIa (TOP2A) inhibitors specifically activate FAS promoter activity in SK-Br3 breast cancer cells. SK-Br3 breast cancer cells growing in 24-well plates were transfected with $300 \mathrm{ng} /$ well of the pGL3-Luciferase construct containing a Luciferase reporter gene driven by an intact 178-bp FAS promoter fragment along with $30 \mathrm{ng} /$ well of the internal control plasmid pRL-CMV, which was used to correct for transfection efficiency. After $18 \mathrm{~h}$, the transfected cells were washed and then incubated in the presence or absence of the antimetabolite drug 5-fluorouracil (5-FU; $10 \mu \mathrm{M})$, the alkylating agent cisplatin (CDDP; $10 \mu \mathrm{M}$ ), the taxane paclitaxel (PTX; $10 \mathrm{nM}$ ), the vinca alkaloid vincristine (VCR; $10 \mathrm{nM}$ ), and the topoisomerase II $\alpha$ (TOP2A) inhibitors doxorubicin (DOX; $1 \mu \mathrm{M}$ ) and etoposide (VP-16; $1 \mu \mathrm{M}$ ). Approximately $24 \mathrm{~h}$ after treatments, luciferase activity from cell extracts was detected using a Luciferase assay system according to the protocol specified by the manufacturer. The magnitude of activation in FAS promoter-Luciferase transfected cells was determined after normalization to the Luciferase activity in cells co-transfected with equivalent amounts of the empty pGL3-Luciferase vector lacking the FAS promoter $(\varnothing$-Luc) and the internal control plasmid pRL-CMV, which was taken as 1.0 -fold. This control value was used to calculate the relative (fold) change in transcriptional activities of FAS promoter following exposure to chemotherapeutic agents after normalization to pRL-CMV activity. Fig. 1a (bottom panel) shows that SK-Br3 cells cultured in the presence of DOX and VP-16 demonstrated a highly significant 2- to 3-fold increase in FAS promoter activity, compared with control cells grown under standard culture conditions. Remarkably, this effect was restricted to TOP2A inhibitors because we failed to observe any significant transcriptional activation of FAS gene promoter following exposure to 5-FU, CDDP, PTX or VCR.

TOP2A inhibitors activate FAS promoter activity in a sterol regulatory element binding protein (SREBP)-independent manner. Since increased FAS expression in cancer cells appears to be part of a more general change in the genetic program controlling lipogenesis $(7,19)$, it could be argued that TOP2A inhibitors-induced activation of FAS gene promoter may represent a general dysregulation of genes coding for other enzymes of the same lipogenic pathway. Indeed, a common up-stream control system based on sterol regulatory element binding proteins (SREBPs)-transcription factors which bind to sterol regulatory elements (SREs) in promoter regions of target genes, operates for the simultaneous transcriptional up-regulation of all the genetic components 
a

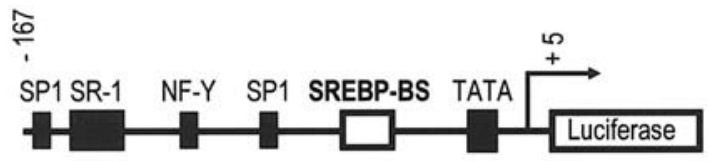

FAS wt-SREBP-BS-Luc

$* P<0.005$ vs. control; Student's $t$ test

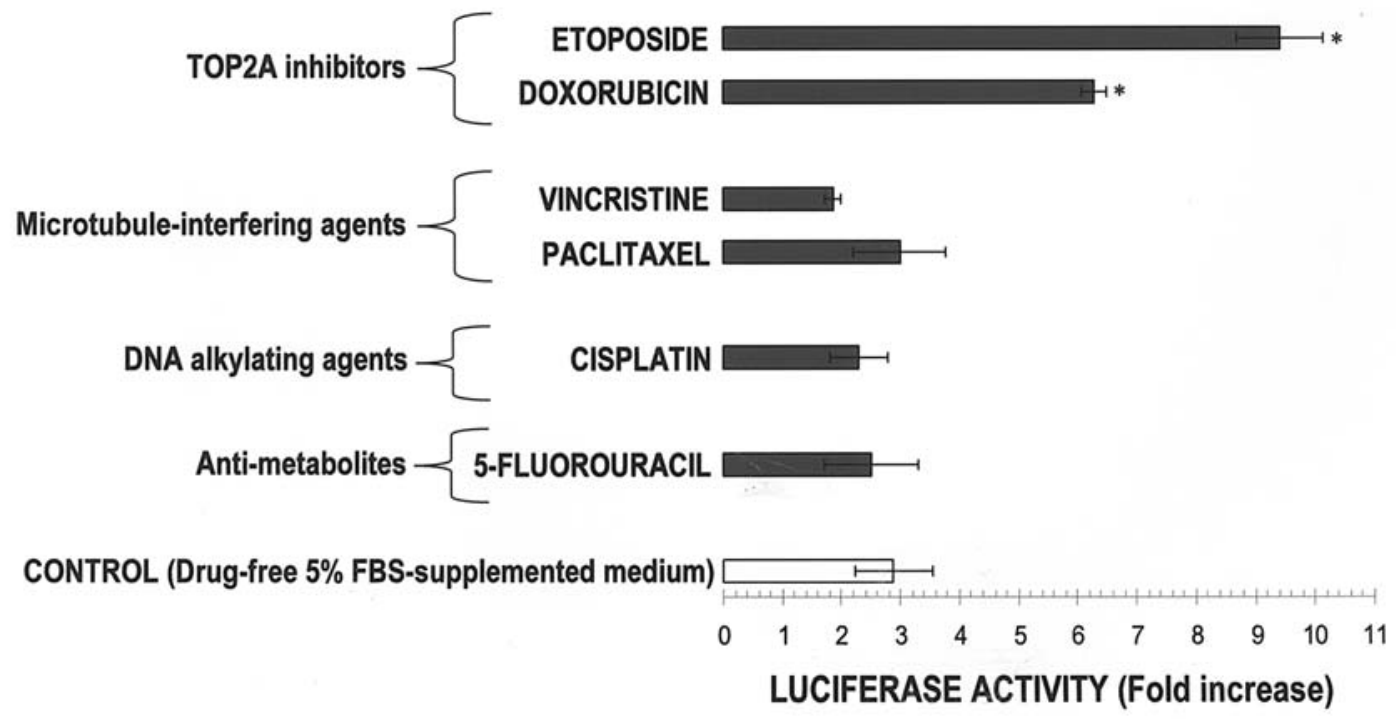

b



FAS $\triangle$ SREBP-BS-LUC

$* \mathrm{P}<0.005$ vs. control; Student's $t$ test

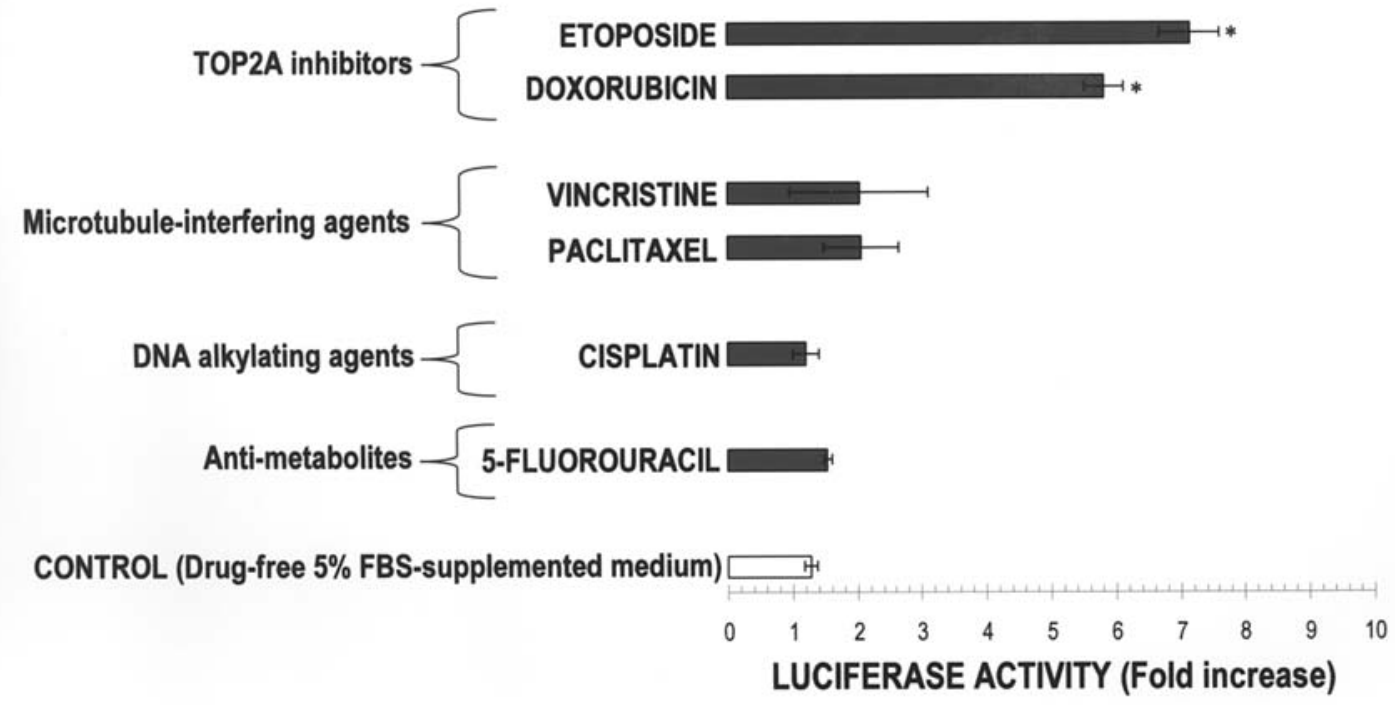

Figure 1. Effects of chemotherapy on the activity of a FAS promoter-reporter construct in SK-Br3 breast cancer cells. SK-Br3 cells were transiently transfected with a plasmid containing a Luciferase reporter gene driven by a 178-bp FAS promoter fragment harboring a well-characterized SREBP-binding site flanked by auxiliary NF-Y and Sp-1 sites (a) or with a similar construct in which the SREBP-binding site was deleted (b). The next day, cultures were treated with chemotherapeutic agents as indicated (see text). After $\sim 24 \mathrm{~h}$ of incubation, cells were lysed. Luciferase activity was measured and relative (fold) changes in transcriptional activities of FAS promoter-Luciferase-transfected cells were calculated. The data are shown as the means (columns) \pm SD (bars) from three separate experiments (performed in duplicate). 


\section{a}
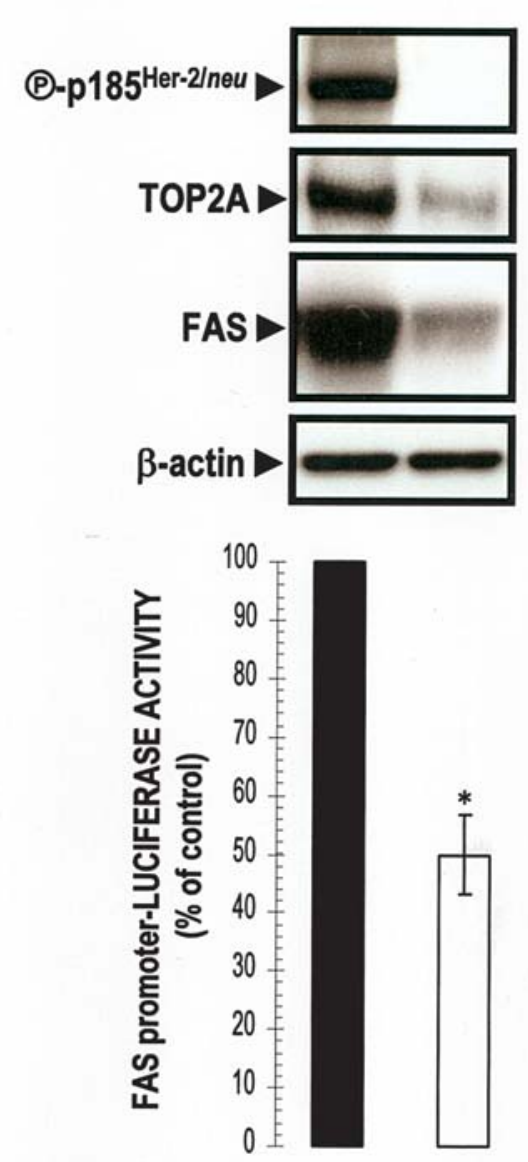

TRASTUZUMAB (10 $\mu \mathrm{g} / \mathrm{ml})$
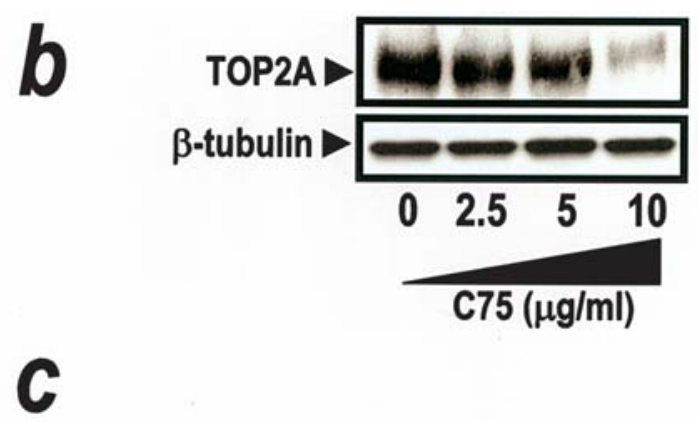

Extracellular matrix

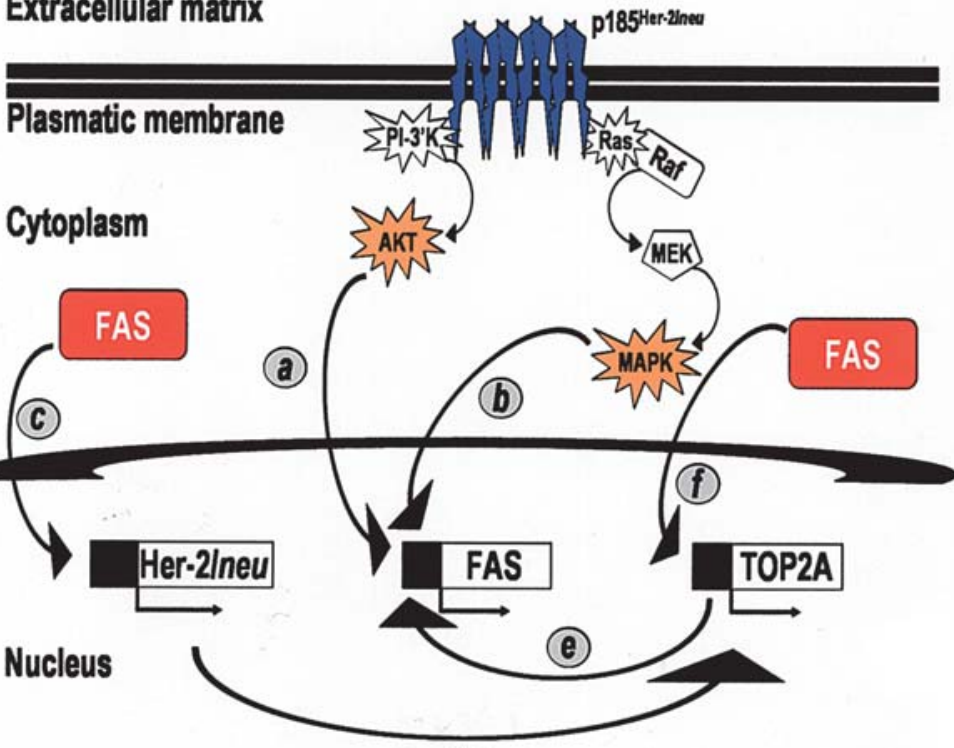

(d)

Figure 2. (a) Her-2/neu signaling regulates TOP2A and FAS expression in SK-Br3 breast cancer cells. Top panels. Representative immunoblot analyses of $\mathrm{P}^{-T y r}{ }^{1248} \mathrm{p} 185^{\mathrm{Her}-2 / n e u}$, TOP2A (nuclear extracts) and FAS proteins in SK-Br3 cells treated with the indicated concentration of trastuzumab for 24 h. B-Actin levels served as a loading control. Bottom panel. SK-Br3 cells were transiently transfected with a plasmid containing a luciferase reporter gene driven by a 178-bp FAS promoter fragment as described in Fig. 1a. The next day, cultures were treated with $10 \mu \mathrm{g} / \mathrm{ml}$ trastuzumab. After $24 \mathrm{~h}$ of incubation, cells were lysed, luciferase activity was measured and relative (fold) changes in transcriptional activities of FAS promoter-luciferase-transfected cells were calculated. The data are shown as the means (columns) \pm SD (bars) from three separate experiments (performed in duplicate). (b) FAS inhibition down-regulates TOP2A protein expression in SK-Br3 breast cancer cells. Representative immunoblot analyses of TOP2A protein (nuclear extracts) in SK-Br3 cells treated with the indicated concentrations of FAS inhibitor C75 for $6 \mathrm{~h}$. B-Tubulin levels served as a loading control. (c) Working model for a novel FAS-Her-2/neu-TOP2A 'functional amplicon' in SK-Br3 breast cancer cells. FAS gene expression in SK-Br3 breast cancer cells is driven by Her-2/neu-triggered signaling, acting in major part through a constitutive downstream activation of the PI-3'K/AKT a) and MAPK ERK1/2 b) transduction cascades and is mediated by cis-acting elements present in the FAS promoter $(10,36)$. FAS activity, in turn, indirectly regulates Her-2/neu gene expression through its ability to modulate PEA3, an Ets transcriptional factor that attenuates Her-2/neu oncogene promoter activity c) (11). Accordingly, Her-2/neu-mediated activation of the FAS promoter and FAS protein levels are markedly decrease upon treatment with inhibitors of Her-2/neu signaling (i.e., trastuzumab, LY294002, U0127). Although Her-2/neu is considered the target gene for the amplification at chromosome band 17q12-21, the amplicon harbors other biologically very relevant genes, such as TOP2A d) $(28,29)$. Our current study reveals that trastuzumab-regulated Her-2/neu signaling also modulates TOP2A protein levels and, concomitantly, FAS promoter activity and FAS protein levels. Moreover, TOP2A signaling appears to specifically regulate FAS promoter e) while FAS activity may indirectly regulate DNA replication in SK-Br3 breast cancer cells through its ability to modulate TOP2A expression f). These findings, altogether, strongly suggest that a tight molecular cross-talk between FAS, Her-2/neu and TOP2A genes 'functional amplicon' is taking place in a subset of breast carcinomas cells.

required for the fatty acid synthesis pathway (20). To examine whether the effects of TOP2A inhibitors DOX and VP-16 on FAS gene activation were mediated by the SBREPbinding site present in the proximal FAS promoter, SK-Br3 cells were transiently transfected with a truncated construct in which the region responsible for SBREP binding is deleted (FAS $\triangle$ SREBP-BS-Luc; Fig. 1b, top panel). The upregulatory effects of TOP2A inhibitors on the transcriptional activation of FAS promoter were not significantly decreased when the FAS promoter was damaged at the SREBP-binding site, while the transcriptional activity of the truncated FAS promoter remained unchanged in response to 5-FU, CDDP,
PTX, and VCR (Fig. 1b, bottom panel). These findings, altogether, reveal for the first time that TOP2A inhibitors, but not other chemotherapeutic agents, can work in an epigenetic fashion by up-regulating the expression of FAS gene in breast cancer cells. FAS promoter activity was not modulated by TOP2A inhibitors or other chemotherapeutic agents in MCF-7 (no Her-2/neu gene amplification; normal copy number of TOP2A gene) and MDA-MB-361 (Her2/neu-amplified; physical deletion of TOP2A gene) (21) beast cancer cell lines (data not shown). The fact that TOP2A inhibitors specifically up-regulate FAS promoter activity in SK-Br3 cells (Her-2/neu-amplified; TOP2A gene 
amplification) (21) strongly suggests that a concurrent overexpression of Her-2/neu and TOP2A is necessary to observe the cross-talk between DOX- and VP-16-induced inhibition of TOP2A and FAS promoter activation.

Trastuzumab-induced blockade of Her-2/neu-driven signaling concomitantly down-regulates the expression of TOP $2 A$ and $F A S$. Considering that FAS inhibition produces profound inhibition of DNA replication and S-phase progression in cancer cells, which suggest a direct linkage at a regulatory level between FAS-catalyzed endogenous fatty acid biogenesis and DNA synthesis in proliferating tumor cells (22-24), we finally asked whether a cross-talk between TOP2A and FAS could exhibit a Her-2/neu-related bi-directional nature. As expected, the anti-Her-2/neu antibody trastuzumab at $10 \mu \mathrm{g} / \mathrm{ml}$, the target plasma level in human studies, (25) led to a decrease

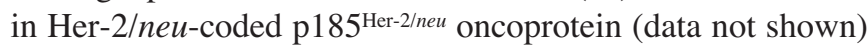
and concomitantly abrogated $\mathrm{p} 185^{\mathrm{Her}-2 / \text { neu}_{\text {-associated }}}$ tyrosine phosphorylation in SK-Br3 breast cancer cells (Fig. 2a) as determined by using a monoclonal Her-2/neu (phosphorspecific) Ab-18 (clone PN2A), which specifically recognizes the activated, tyrosine phosphorylated (P-Tyr ${ }^{1248}$ ) form of

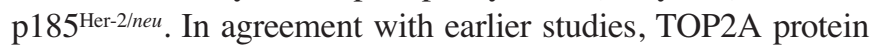
levels decreased during treatment with trastuzumab, in parallel with the decrease in $\mathrm{p} 185^{\mathrm{Her}-2 / \text { neu }}$ receptor phosphorylation (26). Under the same conditions, FAS promoter activity and FAS protein levels were significantly reduced in response to trastuzumab-induced inhibition of Her-2/neu-driven signaling (Fig. 2a).

Pharmacological inhibition of FAS activity down-regulates TOP $2 A$ protein expression. When the expression levels of TOP2A protein were analyzed following a $6 \mathrm{~h}$ exposure of SK$\mathrm{Br} 3$ cells to increasing concentrations of the $\alpha$-methylene- $\gamma$ butyrolactone C75, a novel slow-binding inhibitor of FAS activity (27), a dose-dependent reduction in TOP2A protein expression was observed (Fig. 2b). These results not only suggest that a previously unrecognized bi-directional cross-talk between TOP2A and FAS seems to occur in Her-2/neu-overexpressors but further reveal TOP2A as a good molecular candidate that actively participates in the linkage between FAS-catalyzed endogenous fatty acid metabolism and DNA replication in breast cancer cells $(22,24)$.

\section{Discussion}

TOP2A is a key enzyme in DNA replication and a molecular target for anti-cancer drugs such as DOX and VP-16. The TOP2A gene is located at chromosome band 17q12-q21, adjacent to the Her-2/neu oncogene, which is the most commonly amplified oncogene in breast cancer. Because this physical proximity to Her-2/neu, copy number aberrations also occur in the TOP2A gene. Indeed, TOP2A is either amplified or deleted, with equal frequency, in almost $90 \%$ of Her-2/neu-amplified primary breast tumors $(21,28,29)$. Although all the studies evaluating TOP2A suggest that gene amplification and/or TOP2A protein overexpression might be associated with an increased efficacy of TOP2A inhibitors, the reported studies do not provide the proof of principle needed to authorize the use of TOP2A as a predictive marker for standard practice (30). Our current study indicates that perturbation of TOP2A activity is a novel cellular stress capable of specifically activating FAS promoter in breast cancer cells exhibiting overexpression of Her-2/neu and TOP2A. Therefore, characterization of FAS gene expression may be a clinically valuable molecular surrogate for altered TOP2A activity following TOP2A inhibitors-based chemotherapy in Her-2/neu-overexpressing breast carcinomas. Moreover, our experiments suggest that chemotherapeutic combinations of FAS blockers with TOP2A inhibitors should not be ideal because FAS inhibition leads to decreased target on which TOP2A may act. Accordingly, we previously reported that pharmacological inhibition of FAS activity in combination with anthracyclines, similarly to trastuzumab plus TOP2A inhibitor combinations (26), does not demonstrate synergism in SK-Br3 cells (31).

There have been reports that a region in the long arm of chromosome 17 of SK-Br3 cells spanning 17q22-17q24 (the region to which the human FAS gene has been mapped) is prone to high-level amplification $(32,33)$. However, neither an amplification of the FAS gene nor any gross chromosomal rearrangements have been detected in SK-Br3 breast cancer cells (18). Thus, although FAS gene is not physically located in the Her-2/neu-TOP2A amplicon, our present findings strongly suggest that a tight functional association between FAS, Her-2/neu and TOP2A genes is taking place in a subset of breast carcinomas cells. Therefore, the relationship between Her-2/neu and FAS-dependent endogenous fatty acid metabolism in breast cancer cells is more complex than previously thought, because it involves TOP2A, a key enzyme in the various processes of DNA metabolism, including transcription, recombination, replication, and chromosome segregation during cell division $(34,35)$. Although the clinical and therapeutic importance of FAS status to breast cancer management should be resolved in clinical studies, the characterization of a putative 'functional amplicon' involving FAS, Her-2/neu and TOP2A genes should enhance our understanding of how FASdependent endogenous fatty acid metabolism, once considered a minor anabolic-energy-storage-pathway in normal cells, may be consider a 'metabolic oncogene' that actively contributes to the cancer phenotype through previously unexpected bidirectional cross-talks with well-characterized cancerregulating genes such as Her-2/neu and TOP2A (Fig. 2c).

\section{Acknowledgements}

We wish to thank Johannes Swinnen (Catholic University of Leuven, Leuven, Belgium) for kindly providing the FAS promoter-reporter constructs. Javier A. Menendez is the recipient of a Translational Research Pilot Project (PP2) from the Specialized Program of Research Excellence (SPORE) in Breast Cancer (Robert H. Lurie Comprehensive Cancer Center, Chicago, IL, USA), of a Basic, Clinical and Translational Award (BRCTR0403141) from the Susan G. Komen Breast Cancer Foundation (USA), and of a Breast Cancer Concept Award (BC033538) from the Department of Defense (DOD, USA).

\section{References}

1. Wakil SJ: Fatty acid synthase, a proficient multifunctional enzyme. Biochemistry 28: 4523-4530, 1989. 
2. Kuhajda FP: Fatty-acid synthase and human cancer: new perspectives on its role in tumor biology. Nutrition 16: 202-208, 2000.

3. Kuhajda FP, Jenner K, Wood FD, Hennigar RA, Jacobs LB, Dick JD and Pasternack GR: Fatty acid synthesis: a potential selective target for antineoplastic therapy. Proc Natl Acad Sci USA 91: 6379-6383, 1994.

4. Jensen V, Ladekarl M, Holm-Nielsen P, Melsen F and Soerensen FB: The prognostic value of oncogenic antigen 519 (OA-519) expression and proliferative activity detected by antibody MIB-1 in node-negative breast cancer. J Pathol 176: 343-352, 1995.

5. Alo PL, Visca P, Marci A, Mangoni A, Botti C and Di Tondo U: Expression of fatty acid synthase (FAS) as a predictor of recurrence in stage I breast carcinoma patients. Cancer 77: 474-482, 1996.

6. Alo PL, Visca P, Trombetta G, Mangoni A, Lenti L, Monaco S, Botti C, Serpieri DE and Di Tondo U: Fatty acid synthase (FAS) predictive strength in poorly differentiated early breast carcinomas. Tumori 85: 35-40, 1999.

7. Milgraum LZ, Witters LA, Pasternack GR and Kuhajda FP: Enzymes of the fatty acid synthesis pathway are highly expressed in in situ breast carcinoma. Clin Cancer Res 3: 2115-2120, 1997.

8. Menendez JA, Mehmi I, Verma VA, Teng PK and Lupu R: Pharmacological inhibition of fatty acid synthase (FAS): a novel therapeutic approach for breast cancer chemoprevention through its ability to suppress Her-2/neu (erbB-2) oncogeneinduced malignant transformation. Mol Carcinog 41: 164-178, 2004.

9. Menendez JA, Mehmi I, Atlas E, Colomer R and Lupu R: Novel signaling molecules implicated in tumor-associated fatty acid synthase-dependent breast cancer cell proliferation and survival: role of exogenous dietary fatty acids, p53-p21WAF1/CIP1, ERK1/2 MAPK, p27KIP1, BRCA1, and NF-кB. Int J Oncol 24: 591-608, 2004

10. Kumar-Sinha C, Ignatoski KW, Lippman ME, Ethier SP and Chinnaiyan AM: Transcriptome analysis of HER2 reveals a molecular connection to fatty acid synthesis. Cancer Res 63: 132-139, 2003.

11. Menendez JA, Vellon L, Mehmi I, Oza BP, Ropero S, Colomer R and Lupu R: Inhibition of fatty acid synthase (FAS) suppresses HER2/neu (erbB-2) oncogene overexpression in cancer cells. Proc Natl Acad Sci USA 101: 10715-10720, 2004.

12. Menard S, Pupa SM, Campiglio M and Tagliabue E: Biologic and therapeutic role of HER2 in cancer. Oncogene 22: 6570-6578, 2003.

13. Menendez JA, Lupu R and Colomer R: Inhibition of tumorassociated fatty acid synthase hyperactivity induces synergistic chemosensitization of HER-2/neu-overexpressing human breast cancer cells to docetaxel (taxotere). Breast Cancer Res Treat 84: 183-195, 2004

14. Menendez JA, Colomer R and Lupu R: Inhibition of tumorassociated fatty acid synthase activity enhances vinorelbine (Navelbine)-induced cytotoxicity and apoptotic cell death in human breast cancer cells. Oncol Rep 12: 411-422, 2004.

15. Menendez JA, Vellon L, Colomer R and Lupu R: Pharmacological and small interference RNA-mediated inhibition of breast cancer-associated fatty acid synthase (oncogenic antigen-519) synergistically enhances Taxol ${ }^{\mathrm{TM}}$ (paclitaxel)-induced cytotoxicity. Int J Cancer (In press).

16. Yu D and Hung MC: Role of erbB2 in breast cancer chemosensitivity. Bioessays 22: 673-680, 2000.

17. Thompson BJ, Stern A and Smith S: Purification and properties of fatty acid synthase from a human breast cancer cell line. Biochem Biophys Acta 662: 125-130, 1981.

18. Oskouian B: Overexpression of fatty acid synthase in SKBR3 breast cancer cell line is mediated via a transcriptional mechanism. Cancer Lett 149: 43-51, 2000.

19. Swinnen JV, Vanderhoydonc F, Elgamal AA, Eelen M, Vercaeren I, Joniau S, van Poppel H, Baert L, Goossens K, Heyns W and Verhoeven G: Selective activation of the fatty acid synthesis pathway in human prostate cancer. Int J Cancer 88: 176-179, 2000.
20. Swinnen JV, Ulrix W, Heyns W and Verhoeven G: Coordinate regulation of lipogenic gene expression by androgens: evidence for a cascade mechanism involving sterol regulatory element binding proteins. Proc Natl Acad Sci USA 94: 12975-12980, 1997.

21. Jarvinen TA, Tanner M, Rantanen V, Barlund M, Borg A, Grenman S and Isola J: Amplification and deletion of topoisomerase IIalpha associate with ErbB-2 amplification and affect sensitivity to topoisomerase II inhibitor doxorubicin in breast cancer. Am J Pathol 156: 839-847, 2000.

22. Pizer ES, Chrest FJ, DiGiuseppe JA and Han WF: Pharmacological inhibitors of mammalian fatty acid synthase suppress DNA replication and induce apoptosis in tumor cell lines. Cancer Res 58: 4611-4615, 1998.

23. Li J-N, Gorospe M, Chrest FJ, Kumaravel TS, Evans MK, Han WF and Pizer ES: Pharmacological inhibition of fatty acid synthase activity produces both cytostatic and cytotoxic effects modulated by p53. Cancer Res 61: 1493-1499, 2001.

24. Zhou W, Simpson PJ, McFadden JM, Townsend CA, Medghalchi SM, Vadlamudi A, Pinn ML, Ronnett GV and Kuhajda FP: Fatty acid synthase inhibition triggers apoptosis during S phase in human cancer cells. Cancer Res 63: 7330-7337, 2003.

25. Baselga J, Tripathy D, Mendelsohn J, Baughman S, Benz CC, Dantis L, Sklarin NT, Seidman AD, Hudis CA, Moore J, Rosen PP, Twaddell T, Henderson IC and Norton L: Phase II study of weekly intravenous trastuzumab (Herceptin) in patients with HER2/neu-overexpressing metastatic breast cancer. Semin Oncol 26 (Suppl 12): 78-83, 1999.

26. Harris LN, Yang L, Liotcheva V, Pauli S, Iglehart JD, Colvin OM and Hsieh TS: Induction of topoisomerase II activity after ErbB2 activation is associated with a differential response to breast cancer chemotherapy. Clin Cancer Res 7: 1497-1504, 2001.

27. Kuhajda FP, Pizer ES, Li JN, Mani NS, Frehywot GL and Townsend CA: Synthesis and antitumor activity of an inhibitor of fatty acid synthase. Proc Natl Acad Sci USA 97: 3450-3454, 2000.

28. Jarvinen TA and Liu ET: HER-2/neu and topoisomerase II $\alpha$ in breast cancer. Breast Cancer Res Treat 78: 299-311, 2003.

29. Jarvinen TA and Liu ET: Topoisomerase IIalpha gene (TOP2A) amplification and deletion in cancer-more common than anticipated. Cytopathology 14: 309-313, 2003.

30. Di Leo A and Isola J: Topoisomerase IIalpha as a marker predicting the efficacy of anthracyclines in breast cancer: Are we at the end of the beginning? Clin Breast Cancer 4: 179-186, 2003.

31. Vazquez Martin A, Menendez JA, Ropero S, Montero S, Cortes-Funes $\mathrm{H}$ and Colomer R: Anthracycline resistance on breast cancer cells is reversed by inhibition of fatty acid synthase. Proc Am Assoc Cancer Res 43: 951, 2002.

32. Kallioniemi A, Kallioniemi OP, Piper J, Tanner M, Stokke T, Chen L, Smith HS, Pinkel D, Gray JW and Waldman FM: Detection and mapping of amplified DNA sequences in breast cancer by comparative genomic hybridization. Proc Natl Acad Sci USA 91: 2156-2160, 1994

33. Jayakumar A, Chirala SS, Chinault C, Baldini A, Abu-Elheiga L and Wakil SJ: Isolation and chromosomal mapping of genomic clones encoding the human fatty acid synthase gene. Genomics 23: 420-424, 1994.

34. Osheroff N: DNA topoisomerases. Biochim Biophys Acta 1400: $1-2,1998$.

35. Wang JC: Cellular roles of DNA topoisomerases: a molecular perspective. Nat Rev Mol Cell Biol 3: 430-440, 2002.

36. Menendez JA, Ropero S, Mehmi I, Atlas E, Colomer R and Lupu R: Overexpression and hyperactivity of breast cancerassociated fatty acid synthase (oncogenic antigen-519) is insensitive to normal arachidonic fatty acid-induced suppression in lipogenic tissues but it is selectively inhibited by tumoricidal $\alpha$-linolenic and $\gamma$-linolenic fatty acids: A novel mechanism by which dietary fat can alter mammary tumorigenesis. Int J Oncol 24: 1369-1383, 2004. 